\title{
The effect of preoperative ureteral stenting in retrograde Intrarenal surgery: a multicenter, propensity score-matched study
}

Hyeong Dong Yuk ${ }^{1 *}$, Juhyun Park², Sung Yong Cho ${ }^{1}$, Luck Hee Sung ${ }^{3}$ and Chang Wook Jeong ${ }^{1 *}$ (D)

\begin{abstract}
Background: Stent placement before retrograde intrarenal surgery (RIRS) can theoretically expand the ureter to improve access and remove stones. The purpose of this study was to investigate the effect of preoperative ureteral stenting on access and surgery.

Methods: We retrospectively analyzed patients who underwent RIRS between January 2010 and December 2016 at multiple centers. The patients were divided into two groups based on whether or not a ureteral stent was inserted preoperatively. The characteristics of the stone (size, number, density, and location), the success rate of the access sheath placement, perioperative complications, operative times, hospitalization periods, the period for which the stents remained, postoperative urinary tract infection rates, stone-free rates, and additional treatment rates were analyzed.

Results: Overall, 727 patients were included in the study (113 were pre-stented and 614 were non-stented). The median stone size was $12.2 \mathrm{~mm}$. The overall stone-free rate (SFR) was $85.8 \%$ for the pre-stented group and $83.2 \%$ for the non-stented group, showing no significant $(p=0.498)$ difference between the two groups. Preoperative ureteral stenting improved the success rate of sheath placement ( $93.8 \% \mathrm{vs.} 85.3 \%, p=0.023$ ) during surgery. The access sheath size in participants in the pre-stented group showed a tendency to be larger than that in participants in the non-stented group. However, there were no differences in perioperative complications, operative times, additional treatment rates, and stone-free rates.

Conclusions: Although preoperative ureteral stenting did not affect operative outcomes, it increased the success rate of access sheath placement. Depending on the patient's characteristics, preoperative ureteral stenting can be considered as an adjunctive option when access sheath insertion is considered during RIRS.
\end{abstract}

Keywords: Preoperative ureteral stenting, Renal stone, RIRS, Ureteral access sheath, Ureteral stent, Urolithiasis

* Correspondence: armenia8@snu.ac.kr; drboss@korea.com

'Department of Urology, Seoul National University Hospital, 101 Daehak - ro, Jongno - gu, Seoul 03080, Republic of Korea

Full list of author information is available at the end of the article

C C The Author(s). 2020 Open Access This article is licensed under a Creative Commons Attribution 4.0 International License, which permits use, sharing, adaptation, distribution and reproduction in any medium or format, as long as you give appropriate credit to the original author(s) and the source, provide a link to the Creative Commons licence, and indicate if changes were made. The images or other third party material in this article are included in the article's Creative Commons licence, unless indicated otherwise in a credit line to the material. If material is not included in the article's Creative Commons licence and your intended use is not permitted by statutory regulation or exceeds the permitted use, you will need to obtain permission directly from the copyright holder. To view a copy of this licence, visit http://creativecommons.org/licenses/by/4.0/. The Creative Commons Public Domain Dedication waiver (http://creativecommons.org/publicdomain/zero/1.0/) applies to the data made available in this article, unless otherwise stated in a credit line to the data. 


\section{Background}

Retrograde intrarenal surgery (RIRS) is currently one of the standard treatments for patients with kidney stones $<2 \mathrm{~cm}$ [1]. Advances in technologies such as the development of new flexible ureteroscopes (URS) and small diameter effective lasers have made RIRS an efficient and safe option to manage urinary stones [2]. In stone surgery, a ureteral stent is generally placed after ureteroscopic surgery. It is inserted before ureteroscopic surgery when obstructive uropathy, stone-related complicated infections, or compromised renal function are present [3]. Also, preoperative ureteral stent placement is used when the ureter orifice is too narrow to allow the introduction of the URS.

In the European Association of Urology (EAU) guidelines, the routine placement of ureteral stents prior to RIRS for renal stones is not required. However, several studies have reported that preoperative ureteral stenting affected the outcome of ureteroscopic stone surgery [47]. Therefore, we sought to investigate the relationship between preoperative ureteral stent placement and renal stone surgery outcomes.

\section{Methods}

\section{Study population}

The institutional review board at Seoul National University Hospital and SMG-SNU Boramae Medical Center approved this retrospective study and it was exempted from obtaining informed consent. All research and related protocols used in this study complied with the ethical principles of the Declaration of Helsinki.

We retrospectively analyzed the medical records of 727 consecutive patients with renal stones who underwent RIRS between January 2010 and December 2016 at three institutions. Patients with a previous history of RIRS, ureteroscopic lithotripsy, laparoscopic ureterolithotomy, percutaneous nephrolithotomy (PCNL), and pyeloplasty were excluded from the analysis. Patients with ureteral strictures were also excluded.

\section{Study design}

The patients were divided into two groups, the preoperatively stented and the non-stented groups. The preoperatively stented group underwent preoperative ureteral stent placement as follows: The inserted ureteral stent size was routinely 6-7 Fr. The duration of preoperative ureteral stenting was between one and two weeks. All patients underwent RIRS under general anesthesia and prophylactic second-generation cephalosporin antibiotics were generally administered 1 hour before surgery. The flexible URSs used in RIRS were the Olympus URF-P3, URF-V, and Karl Storz Flex-X2 flexible URSs. In all RIRS procedures, a ureteral access sheath (UAS) was inserted prior to flexible URS insertion. The Guidewires used were Cook Medical Roadrunner $^{\ominus}$ PC Hydrophilic Wire Guide, Terumo Guide Wire Radifocus, and Boston Scientific Amplatz Super Stiff ${ }^{\mathrm{m}}$ Guidewire. The UASs used were the Olympus UroPass Access Sheath (10/12-Fr, 12/14-Fr), the Boston Scientific Navigator ${ }^{\mathrm{rm}}$ Ureteral Access Sheath (11/13-Fr, 12/14-Fr) 4/16-Fr) and the Cook Medical Flexor $^{\oplus}$ (12/14-Fr, 14/16-Fr).

The most commonly used UAS size was $12 / 14 \mathrm{Fr}$. If $12 / 14 \mathrm{Fr}$ size was not suitable, then $11 / 13 \mathrm{Fr}$ and $10 / 12 \mathrm{Fr}$ were used. 14/16-Fr UAS was used once in surgery for patients with kidney stones over $2 \mathrm{~cm}$ and multiple stones. Lumenis ${ }^{\oplus}$ Pulse $^{\mathrm{TM}}$ and VersaPulse ${ }^{\odot}$ PowerSuite $^{\mathrm{TM}}$ $100 \mathrm{~W}$ laser systems were used. As for the ureteral stent, a 6 Fr double J stent was generally used. Laser fiber sizes were 200 and $365 \mu \mathrm{m}$. Laser setting values were long pulse width with $0.4 \sim 2 \mathrm{~J}$ and $10 \sim 40 \mathrm{~Hz}$. Postoperative ureter stents were removed one or two weeks postoperatively. The stone-free rates (SFR) were assessed by computed tomography (CT) scans 3 months after surgery. SFR and clinically insignificant residual fragment (CIRF) were defined according to the diameter of the residual stone at three months after surgery $(0$ $\mathrm{mm}$ and $<4 \mathrm{~mm}$, respectively) as seen on CT [8].

For postoperative pain control, paracetamol and tramadol drugs were used. Antibiotics were maintained for up to one week postoperatively, and anticholinergic drug was administered as needed for ureteral catheter related urinary symptom. Additional treatment was defined as the case where another stone surgery or SWL was performed for residual stone within 3 months after RIRS.

For UAS failure cases, first, only flexible URS along the guidewire was inserted. If the flexible URS insertion was done without access sheath, the laser was then inserted and dusting was performed rather than fragmentation. If the UAS was challenging to insert due to the narrow or kinked ureter, reoperation was performed a week after the ureteral stenting. In some cases, balloon catheter dilation was performed when the flexible URS could not enter after ureteral stenting.

\section{Modified Seoul National University renal stone complexity score (S-ReSC)}

In 2011, our institution devised and validated an S-ReSC scoring system to predict the SFR after PCNL [9]. The $\mathrm{S}-\mathrm{ReSC}$ score is a scoring system that the ratings are calculated by the number of stone involved sites such as the renal pelvis, major calyx, and minor calyx regardless of the size and number of stones. And additional points added for locations where it is difficult to access. Modified S-ReSC was additionally devised and validated to predict SFR after RIRS in 2014. Based on the S-ReSC score, the following groups were obtained: low score 
group, 1-2 points; medium score group, 3-4 points; and high score group, 5-9 points [10].

\section{Statistical analyses}

The characteristics of the stones (location, size, number, density, laterality and complexity), the success rate of the UAS placement, intraoperative complications, operative times, hospitalization durations, ureteral stenting period, postoperative complications, and SFRs were analyzed. In the statistical analyses, the continuous variables are expressed as the mean value and standard deviations. Categorical variables are expressed as the frequencies of events (\%).

Continuous variables are expressed as mean, standard deviation (SD). Baseline characteristics of the two groups were analyzed using Pearson's Chi-squared test and Ttest. Characteristics of renal stones were also analyzed using Pearson's Chi-squared test and T-test. Factors affecting the SFR were analyzed using univariate and multivariate logistic regression. Propensity scorematching was performed to balance potential confounding variables between the preoperatively stented group and the non-stented group to minimize the selection bias. Nearest neighbor 3:1 propensity score-matching was performed. Propensity scores were calculated by preoperative covariates using multivariate logistic regression analysis of each patient. These covariates were age, BMI, sex, creatinine $(\mathrm{Cr})$, glomerular filtration rate (GFR), hydronephrosis, and characteristics of the stone (complexity, size, number, density, and laterality). Balance between the two groups was assessed by absolute standardized differences before and after matching. All statistical tests were performed using IBM SPSS Statistics, version 22.0 (IBM, Armonk, NY, USA) and a $p$-value $<0.05$ indicated statistical significance.

\section{Results}

\section{Clinical characteristics of the patients according to preoperative ureteral stenting}

A total of 727 patients were included in the study, including 614 in the non-stented group (mean age: $55.4 \pm 14.2$ years) and 113 patients (mean age: $57.5 \pm$ 14.1 years) in the preoperatively stented group. No significant differences were seen between the two groups in age, body mass index (BMI), gender, prevalence of diabetes mellitus $(p=0.914)$, hypertension $(p=0.239)$, cardiovascular disease $(p=0.707)$, or cerebrovascular accidents $(p=0.348)$ and preoperative creatinine and hemoglobin levels. However, estimated glomerular filtration rate (eGFR) and hydronephrosis level were significantly $(p=0.002$ and $<0.001)$ lower in the non-stented group than those in the preoperatively stented group. Except for laterality $(p<0.001)$, no significant differences in most stone characteristics, including density, size, and number, were seen between the groups. Modified Seoul National University Renal Stone Complexity scores (S$\mathrm{ReSC}$ ) were used to reflect stone complexity. Complexities of the renal stones were significantly higher in patients in the preoperatively stented group than in the non-stented group and the related hydronephrosis was also different between the two groups. (Table 1).

\section{Perioperative outcomes and complications between the preoperatively stented group and non-stented group}

The access sheath size in participants in the pre-stented group showed a tendency to be larger than that in participants in the non-stented group. Stone-free rates, CIRF, operative times, and additional treatments were not significantly different between the two groups. However, a significant difference was observed in the access sheath placement success rate among patients who underwent preoperative ureteral stenting. An access sheath was successfully inserted in 524 patients (85.3\%) in the non-stented group and in $106(93.8 \%)$ patients in the preoperatively stented group $(p=0.023)$ (Table 2$)$.

Intraoperative and postoperative complications were not associated with preoperative ureteral stenting. Nine grade two or lower complications $(8.0 \%)$ and two grade three or higher complications $(1.8 \%)$ occurred in the preoperatively stented group. Thirty-three grade two or lower complications $(9.7 \%)$ and one grade three or higher complications $(0.4 \%)$ occurred in the non-stented group (Table 3 ). Three (2.7\%) intraoperative complications occurred in the preoperatively stented group, intrarenal bleeding one $(0.9 \%)$, ureteral perforation one $(0.9 \%)$, and arrhythmia one (0.9\%). Forty-seven $(7.6 \%)$ postoperative complication occurred patients in the non-stented group and eight (7.1\%) patients in the preoperatively stented group.

The rate of postoperative infections was similar in both groups. A postoperative UTI and acute pyelonephritis occurred in $25(4.1 \%)$ patients in the non-stented group and five $(4.4 \%)$ in the preoperatively stented group. All 30 postoperative UTI and acute pyelonephritis cases were revisited after discharge and were treated with antibiotics after confirmation with a positive urine test. There was one postoperative sepsis in the nonstented group (Table 3).

\section{Clinical characteristics of the patients and perioperative outcomes and complications in propensity score- matching}

In propensity score-matching between the two groups, no significant differences were seen in patient and stone characteristics between the two groups (Table 1). And perioperative outcomes, such as stone-free rates, clinical stone-free rates, operative times, and hospitalization periods were not significantly different between the two 
Table 1 Baseline characteristics of patients before and after propensity score-matching

\begin{tabular}{|c|c|c|c|c|c|c|}
\hline \multirow[b]{2}{*}{ Variables } & \multicolumn{3}{|c|}{ Before propensity score-matching } & \multicolumn{3}{|c|}{ After propensity score-matching } \\
\hline & $\begin{array}{l}\text { Non-stented } \\
\text { group } \\
(n=614)\end{array}$ & $\begin{array}{l}\text { Preoperatively } \\
\text { stented group } \\
(n=113)\end{array}$ & $\overline{p \text {-value }}$ & $\begin{array}{l}\text { Non-stented } \\
\text { group } \\
(n=339)\end{array}$ & $\begin{array}{l}\text { Preoperatively } \\
\text { stented group } \\
(n=113)\end{array}$ & $p$-value \\
\hline Mean age (year) & $55.4 \pm 14.2$ & $57.5 \pm 14.1$ & 0.151 & $57.6 \pm 13.0$ & $57.5 \pm 14.1$ & 0.949 \\
\hline Mean BMI $\left(\mathrm{Kg} / \mathrm{m}^{2}\right)$ & $24.8 \pm 4.4$ & $24.6 \pm 2.9$ & 0.668 & $24.7 \pm 3.3$ & $24.6 \pm 2.9$ & 0.842 \\
\hline Gender & & & 0.011 & & & 0.594 \\
\hline Male & $386(62.9 \%)$ & $56(49.6 \%)$ & & $183(54.0 \%)$ & $56(49.6 \%)$ & \\
\hline Female & $228(37.1 \%)$ & $57(50.4 \%)$ & & $156(46.0 \%)$ & $57(50.4 \%)$ & \\
\hline Preoperative $\mathrm{Hb}$ & $13.5 \pm 1.8$ & $13.4 \pm 2.0$ & 0.767 & $13.1 \pm 1.8$ & $13.4 \pm 2.0$ & 0.208 \\
\hline Preoperative $\mathrm{Cr}$ & $1.0 \pm 0.6$ & $1.1 \pm 0.5$ & 0.179 & $1.1 \pm 0.8$ & $1.1 \pm 0.5$ & 0.869 \\
\hline Preoperative GFR & $79.9 \pm 28.0$ & $72.0 \pm 24.0$ & 0.002 & $73.1 \pm 22.6$ & $72.0 \pm 24.0$ & 0.724 \\
\hline Modified S-ReSC score group & & & $<0.001$ & & & 0.464 \\
\hline Low & $507(82.6 \%)$ & $28(24.8 \%)$ & & $117(34.5 \%)$ & $28(24.8 \%)$ & \\
\hline Medium & $44(7.2 \%)$ & $13(11.5 \%)$ & & $33(9.7 \%)$ & $13(11.5 \%)$ & \\
\hline High & $63(10.2 \%)$ & $72(63.7 \%)$ & & 189 (55.8\%) & $72(63.7 \%)$ & \\
\hline Laterality & & & $<0.001$ & & & 0.088 \\
\hline Right & $338(55.0 \%)$ & $51(45.1 \%)$ & & $126(37.2 \%)$ & $51(45.1 \%)$ & \\
\hline Left & $276(45.0 \%)$ & $62(54.8 \%)$ & & $213(62.8 \%)$ & $62(54.8 \%)$ & \\
\hline Density of stones (HU) & $823.7 \pm 352.6$ & $889.3 \pm 370.1$ & 0.072 & $908.3 \pm 336.7$ & $889.3 \pm 370.1$ & 0.687 \\
\hline Stone max diameter (mm) & $12.0 \pm 8.5$ & $13.8 \pm 9.7$ & 0.058 & $14.3 \pm 9.9$ & $13.8 \pm 9.7$ & 0.715 \\
\hline Number of stone & & & 0.232 & & & 0.194 \\
\hline 1 & $310(50.5 \%)$ & $49(43.4 \%)$ & & $174(51.3 \%)$ & $49(43.4 \%)$ & \\
\hline 2 & $123(20.0 \%)$ & $30(26.5 \%)$ & & $57(16.8 \%)$ & $30(26.5 \%)$ & \\
\hline$\geq 3$ & $181(29.5 \%)$ & $34(30.1 \%)$ & & $108(31.9 \%)$ & $34(30.1 \%)$ & \\
\hline Hydronephrosis & $169(27.5 \%)$ & 59 (52.2\%) & $<0.001$ & $162(47.8 \%)$ & $59(52.2 \%)$ & 0.425 \\
\hline
\end{tabular}

BMI Body mass index, GFR Glomerular filtration rate, S-ReSC Seoul National University Renal Stone Complexity score, HU Hounsfield units, $H b$ hemoglobin, Cr Creatinine

groups. Intraoperative and postoperative complications were not significantly different between the two groups (Table 2). However, the access sheath placement success rate was higher in patients who underwent preoperative ureteral stenting (Table 2).

\section{Multivariate logistic regression analyses for factors affecting the stone-free rate}

The multivariate regression analyses of factors affecting the SFR showed that stone characteristics, such as size and density of the stone and modified S-ReSC scores were significantly related to stone-free rates (Table 4). Access sheath placement was also significantly related to stone-free rates. In addition to stone size, density, complexity, and access sheath placement were also significant predictors of SFRs after propensity score-matching (Table 4).

\section{Discussion}

In 1987, Bagley first introduced RIRS and reported the results of a flexible RIRS procedure [11]. The development of optical technology, surgical methods, and instruments for RIRS have improved to the point where the procedure is now considered the primary treatment option for patients with kidney stones less than $2 \mathrm{~cm}[1,7,12,13]$. Among the advances that have been made, the access sheath has played a significant role in RIRS. The access sheath allows the flexible URS to quickly and repeatedly enter the kidney and upper ureter and also reduces the risk of injury to the ureter. It also prevents pyelovenous reflux of large amounts of perfusion during surgery [14-18]. The access sheath has been shown to reduce intrarenal pressure and improve vision [14, 19]. Although the usefulness of the access sheath is well known, it is not available for all surgeries. Mogilevkin et al. reported that the failure rate for access sheath placement was approximately $15 \%$ [20]. That study only evaluated cases in which a 14Fr access sheath was inserted and preoperative ureteral stenting was effective. In our study, we observed a $93.8 \%$ success rate for access sheath placement in the preoperatively stented group and $85.3 \%$ in the non-stented group $(p=0.023)$. 
Table 2 Perioperative outcomes according to preoperative ureteral stenting

\begin{tabular}{|c|c|c|c|}
\hline \multirow[b]{2}{*}{ Variables } & \multicolumn{3}{|c|}{ After propensity score-matching } \\
\hline & $\begin{array}{l}\text { Non-stented group } \\
(\mathrm{n}=339)\end{array}$ & $\begin{array}{l}\text { Preoperatively stented group } \\
(n=113)\end{array}$ & $p$-value \\
\hline Operative time (min) & $70.2 \pm 53.0$ & $65.1 \pm 47.4$ & 0.452 \\
\hline Access sheath placement & $297(87.6 \%)$ & $106(93.8 \%)$ & 0.038 \\
\hline Access sheath size (Fr) & & & 0.223 \\
\hline $11 / 13$ & $147(49.5 \%)$ & $49(46.2 \%)$ & \\
\hline $12 / 14$ & $150(50.5 \%)$ & $56(52.8 \%)$ & \\
\hline $14 / 16$ & $0(0.0 \%)$ & $1(0.9 \%)$ & \\
\hline Stone-free rate $(0 \mathrm{~mm})$ & $282(83.2 \%)$ & $97(85.8 \%)$ & 0.498 \\
\hline Clinically insignificant residual fragments $(<4 \mathrm{~mm})$ & $315(92.9 \%)$ & $103(91.2 \%)$ & 0.806 \\
\hline Hospitalization period (days) & $1.4 \pm 1.7$ & $1.3 \pm 0.7$ & 0.279 \\
\hline Additional treatment & $11(3.2 \%)$ & $6(5.3 \%)$ & 0.421 \\
\hline
\end{tabular}

These findings suggest that preoperative ureteral stenting increased the success rate of access sheath placement. Preoperative ureteral stenting is thought to cause passive ureter dilation. A dilated ureter increases the probability of access sheath placement. Several studies have reported that preoperative ureteral stenting affected the outcomes of patients who underwent RIRS [21]. Purlmutter et al. reported that preoperative stents dilated the ureter, passively affecting the outcomes of RIRS [22], while Rubenstein et al. reported that there was a significant effect on the stent and SFR [23]. However,
Fabrizio et al. reported that preoperative ureteral stenting affected the expansion of the ureter but there was no significant correlation with stone clearance [24].

In our study, the SFR was $82.7 \%$ in the non-stented group and $85.8 \%$ in the preoperatively stented group $(p=0.379)$. The CIRF rate was $93.0 \%$ in the non-stented group and $91.2 \%$ in the preoperatively stented group $(p=0.619)$. In propensity score-matching, the SFR was $83.2 \%$ in the non-stented group and $85.8 \%$ in the preoperatively stented group $(p=0.498)$. The CIRF rate was $92.9 \%$ in the non-stented group and $91.2 \%$ in the

Table 3 Perioperative complications according to modified Clavien classification system

\begin{tabular}{|c|c|c|c|}
\hline \multirow[b]{2}{*}{ Variables } & \multicolumn{3}{|c|}{ After propensity score-matching } \\
\hline & $\begin{array}{l}\text { Non-stented group } \\
(\mathrm{n}=339)\end{array}$ & $\begin{array}{l}\text { Preoperatively stented group } \\
(n=113)\end{array}$ & $\overline{p \text {-value }}$ \\
\hline \multicolumn{4}{|l|}{ Intraoperative complication } \\
\hline \multicolumn{4}{|l|}{ Grade I } \\
\hline Intrarenal bleeding & $9(2.7 \%)$ & $1(0.9 \%)$ & 0.410 \\
\hline \multicolumn{4}{|l|}{ Grade II } \\
\hline Arrhythmia & $0(0.0 \%)$ & $1(0.9 \%)$ & 0.845 \\
\hline \multicolumn{4}{|l|}{ Grade III } \\
\hline Ureter perforation & $0(0.0 \%)$ & $1(0.9 \%)$ & 0.872 \\
\hline \multicolumn{4}{|l|}{ Postoperative complication } \\
\hline \multicolumn{4}{|l|}{ Grade I } \\
\hline Fever & $9(2.7 \%)$ & $2(1.8 \%)$ & 0.664 \\
\hline \multicolumn{4}{|l|}{ Grade II } \\
\hline Blood transfusion & $0(0.0 \%)$ & $0(0.0 \%)$ & NA \\
\hline Urinary tract infection & $9(2.7 \%)$ & $5(4.4 \%)$ & 0.519 \\
\hline Acute pyelonephritis & $6(1.8 \%)$ & $0(0.0 \%)$ & 0.478 \\
\hline \multicolumn{4}{|l|}{ Grade III } \\
\hline Ureteral stricture & $1(0.3 \%)$ & $1(0.9 \%)$ & 0.965 \\
\hline \multicolumn{4}{|l|}{ Grade IV } \\
\hline Sepsis & $0(0.0 \%)$ & $0(0.0 \%)$ & NA \\
\hline
\end{tabular}


Table 4 Multivariate logistic regression of stone-free rate predictors

\begin{tabular}{lll}
\hline Variables & \multicolumn{2}{l}{ After propensity score-matching } \\
\cline { 2 - 3 } & OR (95\%Cl) & $\boldsymbol{p}$-value \\
\hline BMI & $0.98(093-1.04)$ & 0.570 \\
Preoperative DJ stenting & $1.07(0.49-2.32)$ & 0.861 \\
Laterality & $0.91(0.43-1.93)$ & 0.805 \\
Number of stones & $0.92(0.78-1.09)$ & 0.348 \\
Stone max diameter & $0.95(0.90-0.99)$ & 0.028 \\
Density of stones & $0.99(0.99-1.00)$ & 0.007 \\
Modified S-ReSC score & $0.42(0.29-0.75)$ & 0.002 \\
Hydronephrosis & $0.91(0.46-1.79)$ & 0.781 \\
Access sheath placement & $1.27(1.08-1.88)$ & 0.029 \\
\hline
\end{tabular}

BMI Body mass index, S-ReSC Seoul National University Renal Stone Complexity score

preoperatively stented group $(p=0.806)$. These results indicate that preoperative ureteral stenting was not significantly associated with stone clearance. In multivariate logistic regression, stone characteristics such as size, density, and complexity affected the stone-free rate. In addition to stone characteristics, access sheath placement has been shown to affect the stone-free rate. Propensity score- matching results also showed that stone size, density, complexity, and access sheath placement were important predictors of SFRs (Table 4). However, preoperative ureteral stenting had no significant effect on SFRs. Most RIRS surgeries are performed without preoperative ureteral stenting. In general, preoperative ureteral stenting is considered when UAS insertion is difficult or when it is difficult to insert flexible URS directly. Although ureteral preoperative stenting did not affect SFR, it increased the success rate of UAS insertion. This might be an important source for the prediction of patients who need preoperative stenting in the future. Large-scale prospective studies related to the prediction of patients who need preoperative stents need to be performed in the future.

In our study, patients in the preoperatively stented group had fewer overall complications than the nonstented group, although the difference between the two groups was not statistically significant (Grade I-II complication rates: 33 (9.7\%) versus 9 (8.0\%); grade IIIIV complication rates: $0.3 \%$ versus $1.8 \%$ ) (Table 3 ). One patient $(0.9 \%)$ developed ureter perforation who was a preoperatively stented patient. In a previous study, patients in the pre-stented groups had fewer complications than those in the non-stented group (major complications: $0.6 \%$ vs. $1.6 \%$; minor complications: $4.7 \%$ vs. $9.4 \%$ ) [4]. Ureter perforation was also relatively lower in the pre-stented group $(2.7 \%$ vs. $9.4 \%)$ [4]. Similar to our results, Rubenstein et al. have also shown no significant difference in the rate of complications between the two groups [23]. Lee et al. have compared a short preopstenting group, a long preop-group, and a no-stenting group and found no significant difference in overall complication among the three groups [25].

We acknowledge that our study had limitations. First, we used a retrospective study design. Second, this study was not free from selection bias due to the use of multiple laser systems and laser fibers, multiple types and sizes of ureteric access sheathes, multiple types of URS, and multiple surgeons. Third, we did not insert the preoperative stents randomly, therefore, a selection bias could have occurred. Patients in the preoperatively stented group had more complex stone positions of than those in the non-stented group and the degree of hydronephrosis was higher in this group. Fourth, UAS is not a routine practice in flexible URS. Some endourologists prefer to go directly with a flexible URS over a guidewire. Therefore, propensity score-matching was performed for calibration. If these limitations are addressed in future studies, the results are expected to be more significant.

\section{Conclusions}

This study showed that preoperative ureteral stenting increased the success rate of access sheath placement. However, preoperative stenting had no significant effect on operative outcomes or complications such as SFRs, operative times, perioperative complications, or ureteral strictures.

\section{Abbreviations}

BMI: Body mass index; CT: Computed tomography; eGFR: Estimated glomerular filtration rate; HU: Hounsfield units; RIRS: Retrograde intrarenal surgery; S-ReSC: Seoul National University Renal Stone Complexity score; UTI: Urinary tract infection

\section{Acknowledgements}

Not applicable.

\section{Consent to publication}

Not applicable.

\section{Authors' contributions}

$H Y, J P, S C$, LS and CJ contributed to the concept and project planning. HY, SC, and CJ contributed significantly to the writing of this paper. CJ contributed significantly to the editing of the final draft. The authors read and approved the final manuscript.

\section{Funding}

Not applicable.

\section{Availability of data and materials}

The datasets used and/or analysed during the current study are available from the corresponding author upon reasonable request.

\section{Ethics approval and consent to participate}

The study was approved by the institutional review board at Seoul National University Hospital and SMG-SNU Boramae Medical Center (H1710-036-891). We conducted a retrospective case study and were exempted from obtaining prior consent of the patients. The research protocol was in accordance with the Declaration of Helsinki guidelines. This study is involved at human 
participants. However, retrospective patient case studies have exempted patient's informed consent.

\section{Competing interests}

The authors declare that they have no competing interests.

\section{Author details}

'Department of Urology, Seoul National University Hospital, 101 Daehak - ro, Jongno - gu, Seoul 03080, Republic of Korea. ${ }^{2}$ Department of Urology, Asan Medical Center, University of Ulsan College of Medicine, Seoul, South Korea. ${ }^{3}$ Department of Urology, Inje University Sanggye Paik Hospital, 1342

Dongil-ro, Nowon-gu, Seoul 01757, South Korea.

Received: 20 April 2019 Accepted: 4 September 2020

Published online: 14 September 2020

\section{References}

1. Bultitude M, Smith D, Thomas K. Contemporary Management of Stone Disease: the new EAU Urolithiasis guidelines for 2015. Eur Urol. 2016;69: 483-4.

2. Breda A, Emiliani E, Millan F, et al. The new concept of ureteral access sheath with guidewire disengagement: one wire does it all. World J Urol. 2016;34:603-6

3. Joshi HB, Stainthorpe A, MacDonagh RP, Keeley FX Jr, Timoney AG, Barry MJ. Indwelling ureteral stents: evaluation of symptoms, quality of life and utility. J Urol. 2003:169:1065-9 discussion 9.

4. Lumma PP, Schneider P, Strauss A, et al. Impact of ureteral stenting prior to ureterorenoscopy on stone-free rates and complications. World J Urol. 2013; 31:855-9.

5. Kawahara $\mathrm{T}$, Ito $\mathrm{H}$, Terao $\mathrm{H}$, et al. Preoperative stenting for ureteroscopic lithotripsy for a large renal stone. Int J Urol. 2012;19:881-5.

6. Chu L, Sternberg KM, Averch TD. Preoperative stenting decreases operative time and reoperative rates of ureteroscopy. J Endourol. 2011;25:751-4.

7. Miernik A, Wilhelm K, Ardelt PU, Adams F, Kuehhas FE, Schoenthaler M. Standardized flexible ureteroscopic technique to improve stone-free rates. Urology. 2012;80:1198-202.

8. Osman MM, Alfano Y, Kamp S, Haecker A, Alken P, Michel MS, Knoll T. 5year-follow-up of patients with clinically insignificant residual fragments after extracorporeal shockwave lithotripsy. Eur Urol. 2005;47(6):860-64. https://www.nature.com/articles/ncpuro0239\#article-info.

9. Jeong CW, Jung JW, Cha WH, Lee BK, Lee S, Jeong SJ, et al. Seoul National University renal stone complexity score for predicting stone-free rate after percutaneous Nephrolithotomy. PLoS One. 2013;8(6):e65888.

10. Jung JW, Lee BK, Park YH, Lee S, Jeong SJ, Lee SE, et al. Modified Seoul National University renal stone complexity score for retrograde intrarenal surgery. Urolithiasis. 2014:42(4):335-40.

11. Bagley DH, Huffman JL, Lyon ES. Flexible ureteropyeloscopy: diagnosis and treatment in the upper urinary tract. J Urol. 1987:138:280-5.

12. Wang AJ, Preminger GM. Modern applications of ureteroscopy for intrarenal stone disease. Curr Opin Urol. 2011:21:141-4.

13. Desai MR, Ganpule A. Flexible ureterorenoscopy. BJU Int. 2011;108:462-74.

14. Ng YH, Somani BK, Dennison A, Kata SG, Nabi G, Brown S. Irrigant flow and intrarenal pressure during flexible ureteroscopy: the effect of different access sheaths, working channel instruments, and hydrostatic pressure. J Endourol. 2010;24:1915-20.

15. Stern JM, Yiee J, Park S. Safety and efficacy of ureteral access sheaths. J Endourol. 2007:21:119-23.

16. L'Esperance JO, Ekeruo WO, Scales CD Jr, et al. Effect of ureteral access sheath on stone-free rates in patients undergoing ureteroscopic management of renal calculi. Urology. 2005;66:252-5.

17. Monga M, Bodie J, Ercole B. Is there a role for small-diameter ureteral access sheaths? Impact on irrigant flow and intrapelvic pressures. Urology. 2004;64: 439-41 discussion 41-2.

18. Rehman J, Monga M, Landman J, et al. Characterization of intrapelvic pressure during ureteropyeloscopy with ureteral access sheaths. Urology. 2003:61:713-8

19. Somani BK, Aboumarzouk O, Srivastava A, Traxer O. Flexible ureterorenoscopy: tips and tricks. Urol Ann. 2013:5:1-6.

20. Mogilevkin Y, Sofer M, Margel D, Greenstein A, Lifshitz D. Predicting an effective ureteral access sheath insertion: a bicenter prospective study. J Endourol. 2014;28:1414-7.
21. Shields JM, Bird VG, Graves R, Gomez-Marin O. Impact of preoperative ureteral stenting on outcome of ureteroscopic treatment for urinary lithiasis. J Urol. 2009;182:2768-74.

22. Perlmutter AE, Talug C, Tarry WF, Zaslau S, Mohseni H, Kandzari SJ. Impact of stone location on success rates of endoscopic lithotripsy for nephrolithiasis. Urology. 2008;71:214-7.

23. Rubenstein RA, Zhao LC, Loeb S, Shore DM, Nadler RB. Prestenting improves ureteroscopic stone-free rates. J Endourol. 2007;21:1277-80.

24. Fabrizio MD, Behari A, Bagley DH. Ureteroscopic management of intrarenal calculi. J Urol. 1998;159:1139-43.

25. Lee MH, Lee IJ, Kim TJ, Lee SC, Jeong CW, Hong SK, et al. The effect of short-term preoperative ureteral stenting on the outcomes of retrograde intrarenal surgery for renal stones. World J Urol. 2019;37:1435-40.

\section{Publisher's Note}

Springer Nature remains neutral with regard to jurisdictional claims in published maps and institutional affiliations.

Ready to submit your research? Choose BMC and benefit from:

- fast, convenient online submission

- thorough peer review by experienced researchers in your field

- rapid publication on acceptance

- support for research data, including large and complex data types

- gold Open Access which fosters wider collaboration and increased citations

- maximum visibility for your research: over $100 \mathrm{M}$ website views per year

At $\mathrm{BMC}$, research is always in progress.

Learn more biomedcentral.com/submissions 